\title{
Baixas Doses de Bupivacaína a 0,5\% Isobárica para Raquianestesia Unilateral *
}

\section{Low Isobaric 0.5\% Bupivacaine Doses for Unilateral Spinal Anesthesia}

\author{
Luiz Eduardo Imbelloni, TSA ${ }^{1}$; Lúcia Beato, TSA ${ }^{2}$; M.A. Gouveia, TSA ${ }^{3}$
}

\author{
RESUMO \\ Imbelloni LE, Beato L, Gouveia MA - Baixas Doses de Bupiva- \\ caína a $0,5 \%$ Isobárica para Raquianestesia Unilateral
}

JUSTIFICATIVA E OBJETIVOS: A raquianestesia unilateral pode apresentar diversas vantagens, principalmente no paciente ambulatorial. Baixas doses de anestésicos locais, lenta velocidade de injeção subaracnóidea e posição de decúbito lateral são relacionados com a facilidade de produzir distribuição unilateral na raquianestesia. Neste estudo, foi verificada a possibilidade de se obter raquianestesia unilateral com bupivacaína a 0,5\% isobárica.

MÉTODO: A raquianestesia com $1 \mathrm{ml}$ de bupivacaína isobárica a 0,5\% (5 mg) foi realizada através de agulha 27G Quincke em 25 pacientes, estado físico ASA I e II submetidos à cirurgias ortopédicas. A punção subaracnóidea foi realizada por via lateral com o paciente em decúbito lateral, com o membro a ser operado voltado para cima, e $1 \mathrm{ml}$ de bupivacaína isobárica foi injetado na velocidade de $1 \mathrm{ml} .30 \mathrm{~s}^{-1}$. Bloqueios sensitivo e motor (picada de agulha e escala de 0 a 3) foram comparados entre os lados a ser operado e o contralateral.

RESULTADOS: Os bloqueios motor e sensitivo entre o lado operado e o contralateral foram significativamente diferentes em todos os tempos avaliados. No membro operado todos os pacientes apresentaram anestesia cirúrgica. No membro contralateral, aos 20 minutos, 9 pacientes apresentaram bloqueio sensitivo; aos 40 minutos 18 pacientes e aos 60 minutos 17 pacientes. Portanto, o bloqueio sensitivo unilateral ocorreu em 7 pacientes (28\%) e em ambos os membros em 18 pacientes (72\%). Raquianestesia unilateral foi obtida em $28 \%$ dos pacientes. Estabilidade hemodinâmica foi observada em todos os pacientes. Nenhum paciente desenvolveu cefaléia pós-punção dura-máter.

CONCLUSÕES: A bupivacaína isobárica (5 mg) proporciona predominante bloqueio unilateral após 20 minutos na posição lateral. A solução isobárica de bupivacaína se mobiliza dentro do LCR após 20 minutos, resultando num bloqueio unilateral em apenas $28 \%$ dos pacientes. A principal vantagem da raquianestesia unilateral é a estabilidade hemodinâmica e sua

\footnotetext{
* Recebido da (Received from) Casa de Saúde Santa Maria, Clínica São Bernardo e Hospital IASERJ, Rio de Janeiro, RJ

1. Diretor do Instituto de Anestesia Regional, Anestesiologista da Casa de Saúde Santa Maria e Clínica São Bernardo. Rio de Janeiro

2. Anestesiologista da Casa de Saúde Santa Maria e Clínica São Bernardo. Rio de Janeiro

3. Diretor do Instituto de Anestesia Regional, Chefe do Serviço de Anestesiologia do Hospital Central do IASERJ, Rio de Janeiro
}

Apresentado (Submitted) em 30 de maio de 2003

Aceito (Accepted) para publicação em 18 de agosto de 2003

Endereço para correspondência (Correspondence to)

Dr. Luiz Eduardo Imbelloni

Av. Epitácio Pessoa, 2356/203 Lagoa

22471-000 Rio de Janeiro, RJ

E-mail: imbelloni@openlink.com.br

(c) Sociedade Brasileira de Anestesiologia, 2004 rápida regressão, podendo ser uma nova opção para cirurgia ambulatorial.

Unitermos: ANESTÉSICOS, Local, bupivacaína; TÉCNICAS ANESTÉSICAS, Regional, subaracnóidea

\section{SUMMARY}

Imbelloni LE, Beato L, Gouveia MA - Low Isobaric 0.5\% Bupivacaine Doses for Unilateral Spinal Anesthesia

BACKGROUND AND OBJECTIVES: Unilateral spinal anesthesia may be advantageous, especially in the outpatient setting. Low local anesthetic doses, slow spinal injection rate and the lateral position have been related to the easiness of inducing unilateral spinal anesthesia. This study aimed at investigating the possibility of inducing unilateral spinal anesthesia with isobaric $0.5 \%$ bupivacaine.

METHODS: Spinal anesthesia with $1 \mathrm{~mL}$ isobaric $0.5 \%$ bupivacaine (5 mg) was induced through 27G Quincke needle in 25 physical status ASA I and II patients undergoing orthopedic procedures. Dural puncture was performed with patients in lateral decubitus with the limb to be operated facing upwards. One mililiter of isobaric bupivacaine was injected in 30 seconds. Sensory and motor blocks were compared between the operated and the contralateral side.

RESULTS: Motor and sensory blocks on operated and contralateral sides were significantly different in all studied moments. All patients presented surgical anesthesia in the operated limb. At 20 minutes, nine patients presented sensory block in the contralateral side; at 40 minutes they were 18 and at 60 minutes they were 17 patients. So, unilateral block was present in seven patients (28\%) and bilateral block was present in the remaining 18 patients $(72 \%)$. No patient developed post-dural puncture headache.

CONCLUSIONS: Isobaric bupivacaine $(5 \mathrm{mg})$ provides predominant unilateral block after 20 minutes in the lateral position. Isobaric bupivacaine moves in the CSF after 20 minutes resulting in just $28 \%$ unilateral blocks. Major advantages of unilateral spinal anesthesia are hemodynamic stability and short duration and it might be a new alternative for outpatient procedures.

Key Words: ANESTHETICS, Local, bupivacaine; ANESTHETIC TECHNIQUES, Regional: spinal block

\section{INTRODUÇÃO}

$\mathrm{D}$ versos anestesiologistas se deparam diariamente com procedimentos cirúrgicos envolvendo apenas um membro inferior, principalmente em cirurgias ambulatoriais e ortopédicas. Nestas situações a raquianestesia unilateral apresenta algumas vantagens sobre a raquianestesia convencional. Essas vantagens incluem a redução da incidência de hipotensão arterial durante a raquianestesia ${ }^{1-3}$, recuperação mais rápida da anestesia ${ }^{2,3}$ e aumento da satisfação ${ }^{2,3}$. O decúbito lateral, baixas doses de anestésicos, direção da agulha ponta de lápis e lenta velocidade de 
injeção têm sido sugeridos como facilitadores na realização de raquianestesia unilateral ${ }^{4,5}$. Teoricamente, injeções de soluções não isobáricas de anestésicos locais podem produzir bloqueio unilateral no paciente na posição de decúbito lateral. Soluções isobáricas de bupivacaína foram comparadas com soluções hiperbáricas ${ }^{6}$ e hipobáricas ${ }^{7}$ com objetivo de se obter raquianestesia unilateral.

O objetivo desta investigação foi avaliar a tentativa de distribuição assimétrica do bloqueio entre o membro a ser operado e o contralateral e a influência na incidência de hipotensão arterial durante a raquianestesia para procedimentos ortopédicos de membros inferiores com baixas doses de bupivacaína a $0,5 \%$ isobárica.

\section{MÉTODO}

Após aprovação da Diretoria de Publicação e Divulgação das Clínicas e consentimento informado, participaram deste estudo prospectivo 25 pacientes estado físico ASA I e II, com idades entre 21 e 67 anos, submetidos à raquianestesia para cirurgias ortopédicas eletivas e de urgências, envolvendo apenas um dos membros. Critérios de exclusão foram hipovolemia, distúrbios de coagulação, infecção e recusa do método proposto. A monitorização incluiu avaliação da pressão arterial por método não invasivo, freqüência cardíaca e oximetria de pulso. Não foi administrada nenhuma medicação pré-anestésica. Após a chegada à sala de operação, foi instalada venóclise com solução de Ringer com lactato e injetados 25 a $50 \mu \mathrm{g}$ de fentanil.

O paciente foi colocado em decúbito lateral com o membro a ser operado voltado para cima. Acoluna vertebral foi posicionada horizontalmente antes da punção subaracnóidea e a mesa nivelada na posição horizontal (zero) durante o procedimento. A punção subaracnóidea foi realizada no espaço $\mathrm{L}_{3}-\mathrm{L}_{4}$ com agulha $27 \mathrm{G}$ tipo Quincke (B. Braun) pela via paramediana e injetados $5 \mathrm{mg}$ de bupivacaína isobárica a $0,5 \%$ na velocidade de $1 \mathrm{ml} .30 \mathrm{~s}^{-1} \mathrm{com}$ a ponta orientada para o membro superior. O paciente foi mantido nesta posição por 20 minutos e em seguida colocado em decúbito dorsal horizontal.

O nível sensitivo foi avaliado pela perda da sensação de frio (gelo) e picada de agulha $27 \mathrm{G}$, enquanto que o bloqueio motor foi avaliado pela escala modificada de Bromage ( $0=\mathrm{sem}$ bloqueio motor a 3 = bloqueio motor completo). A avaliação dos bloqueios sensitivo e motor foi realizada em ambos os membros aos 20, 40 e 60 minutos e ao final da cirurgia. Os parâmetros hemodinâmicos foram avaliados a cada três minutos durante os primeiros 10 minutos após a punção subaracnóidea e posteriormente a cada cinco minutos até o final da cirurgia.

A hipotensão arterial foi definida como a diminuição de $30 \%$ dos valores basais, enquanto que bradicardia foi definida como FC menor do $50 \mathrm{bpm}$. A hipotensão arterial seria tratada com $1 \mathrm{mg}$ de etilenofenilefrina e bradicardia com $0,5 \mathrm{mg}$ de atropina, por via venosa. Durante o procedimento cirúrgico um garrote pneumático foi inflado $100 \mathrm{mmHg}$ acima da pressão arterial sistólica no membro a ser operado. Um fluxo de
21. $\mathrm{min}^{-1}$ de oxigênio foi administrado através de cateter nasal ou máscara de Hudson. Midazolam (1 a $2 \mathrm{mg}$ ) foi administrado após a segunda avaliação (40 minutos). Os pacientes foram acompanhados até o terceiro dia de pós-operatório para se obter informação sobre sua satisfação com a técnica, para pesquisa de cefaléia pós-punção ou sintomas neurológicos transitórios (SNT), e até o $30^{\circ}$ dia com relação às complicações neurológicas definitivas.

As variáveis quantitativas foram analisadas, em termos de estatística descritiva, com apresentação de média \pm desvio padrão, mínimo, máximo e mediana - quando foi a melhor alternativa, sendo as comparações dos bloqueios nos dois membros pelo teste não paramétrico do sinal e as correlações avaliadas pelo coeficiente de correlação de Pearson. $O$ nível de significância adotado foi de 5\%.

\section{RESULTADOS}

Os dados demográficos dos pacientes estão apresentados na tabela I.

Tabela I - Dados Demográficos, Duração da Cirurgia e do Bloqueio Sensitivo

\begin{tabular}{lc}
\hline Idade (anos) * & $39,11 \pm 13,75$ \\
Feminino & $43,90 \pm 14,50$ \\
Masculino & $33,40 \pm 11,40$ \\
Peso $(\mathrm{kg})^{*}$ & $67,12 \pm 13,59$ \\
Altura $(\mathrm{cm})^{*}$ & $163,44 \pm 6,60$ \\
Sexo & 14 \\
Feminino & 11 \\
$\quad$ Masculino & $1,33 \pm 0,19$ \\
Duração da cirurgia $(\mathrm{h}){ }^{*}$ & \\
Duração do bloqueio $(\mathrm{h}){ }^{*}$ & $2,34 \pm 0,33$ \\
$\quad$ Membro operado & $1,64 \pm 0,67$ \\
Membro contralateral &
\end{tabular}

*Valores expressos pela Média \pm DP

O bloqueio unilateral foi observado em $64 \%$ dos pacientes aos 20 minutos. Aos 40 e 60 minutos apenas $28 \%$ dos pacientes apresentaram bloqueio unilateral. No final da cirurgia, $32 \%$ dos pacientes apresentaram bloqueio unilateral. A regressão completa do bloqueio no membro operado ocorreu em média, com $2,34 \pm 0,33$ h e no membro contralateral com $1,64 \pm 0,67 \mathrm{~h}$. Não houve diferença significante na regressão completa do bloqueio quanto ao sexo (valor- $p=0,95$, operado; valor- $p=0,97$, contralateral), nem na duração da cirurgia (valor- $p=0,86$ ), com as mulheres com maior idade média que os homens (valor- $p=0,028$ )

Adispersão do bloqueio sensitivo no membro operado variou de $T_{8}$ a $L_{1}$ e no membro contralateral de $T_{12}$ a $L_{3}$, mostrando uma nítida dissociação entre os membros. No membro contralateral aos 20 minutos 9 pacientes apresentaram bloqueio sensitivo, aos 40 minutos 18 pacientes e aos 60 minutos 17 pacientes. Portanto, o bloqueio sensitivo unilateral ocorreu em 7 pacientes (28\%) e em ambos os membros em 18 paci- 
entes $(72 \%)$. O bloqueio no membro operado foi estatisticamente maior (valores- $p<0,0001$ avaliados pelo teste do sinal), como ilustram as linhas pontilhadas nas figuras 1 a 4 . Há correlação linear entre as dispersões do bloqueio sensitivo nos dois membros, conforme ilustram as figuras 1 a 4 .

No membro operado, aos 20 minutos o bloqueio motor completo (grau 3) ocorreu em 21 pacientes e o grau 2 em 4 pacientes. Aos 40 minutos, mais dois pacientes atingiram o bloqueio motor completo. No membro contralateral, ocorreu bloqueio motor completo aos 20 minutos em apenas 1 paciente, grau 2 em 5, grau 1 em 3 e ausente em 16. Aos 40 minutos ocorreu bloqueio motor completo em 2 pacientes, grau $2 \mathrm{em}$ 12, grau 1 em 5 e ausência de bloqueio em 6 (Figura 5 ). O bloqueio motor unilateral ocorreu em 16 pacientes $(64 \%)$ aos 20 minutos em 6 pacientes (24\%) aos 40 minutos. O bloqueio motor no membro operado foi maior que no membro contralateral em cada avaliação (valores $p<0,0001$ avaliado pelo teste do sinal).

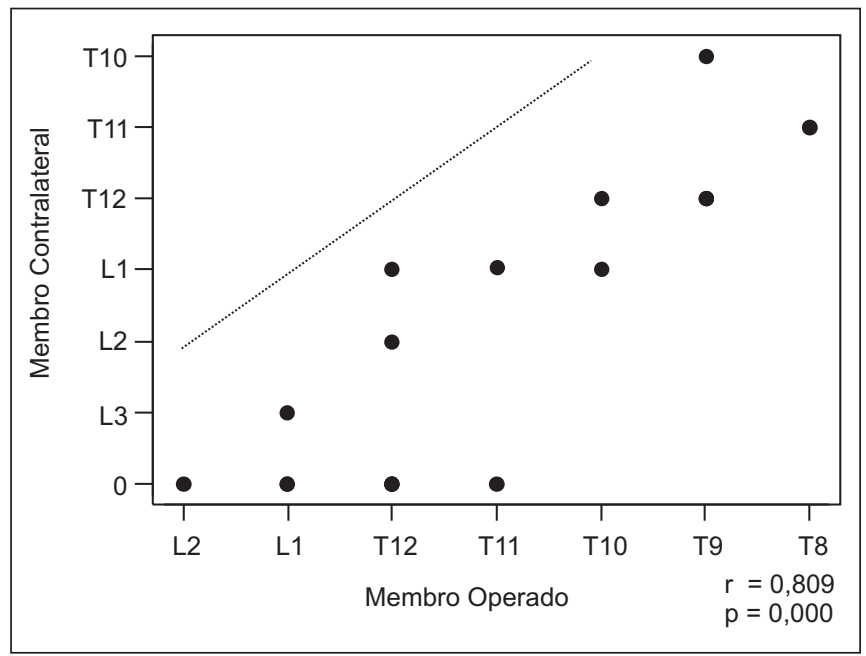

Figura 1- Dispersão do Bloqueio Sensitivo aos 20 Minutos: Membro Operado Versus Membro Contralateral

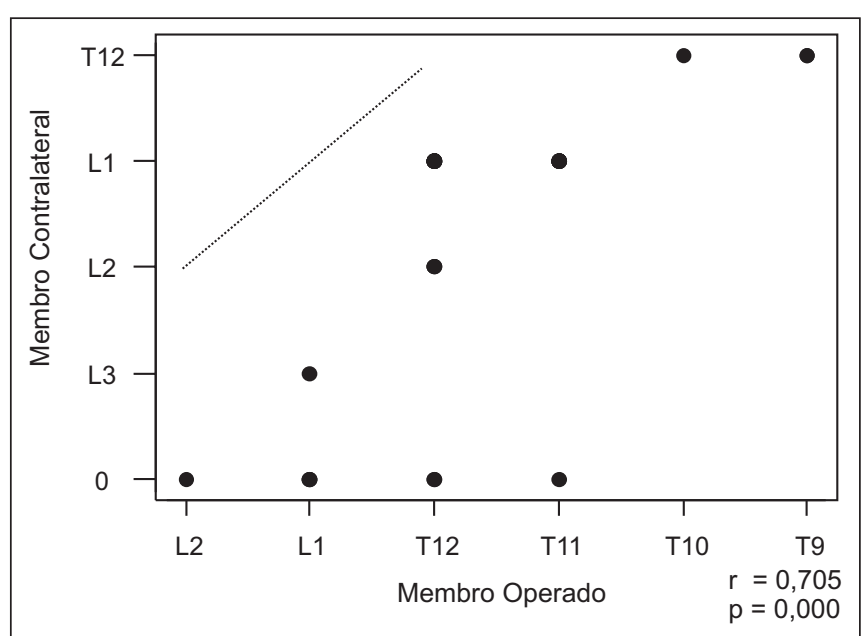

Figura 2 - Dispersão do Bloqueio Sensitivo aos 40 Minutos: Membro Operado Versus Membro Contralateral

Revista Brasileira de Anestesiologia

Vol. 54, № 3, Maio - Junho, 2004

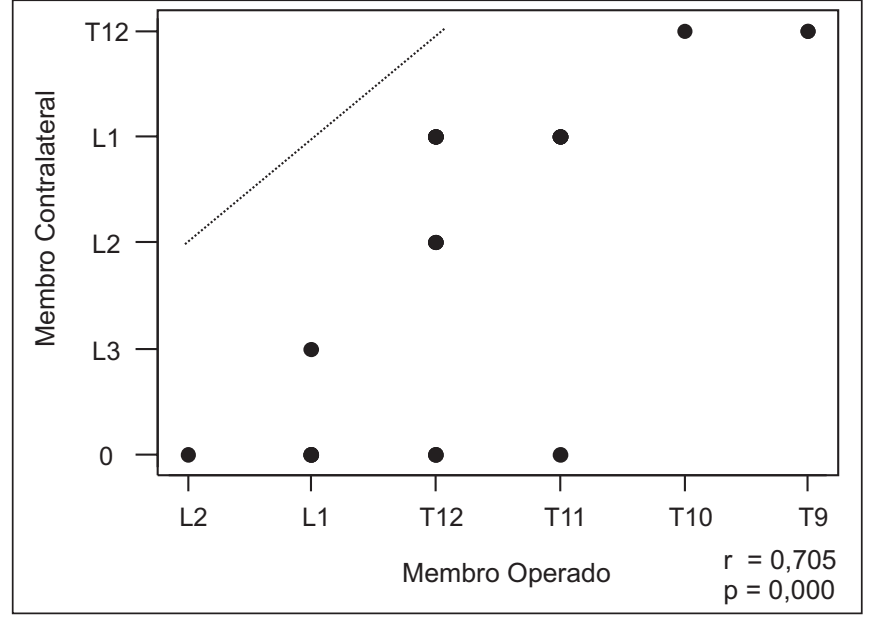

Figura 3 - Dispersão do Bloqueio Sensitivo aos 60 minutos: Membro Operado Versus Membro Contralateral

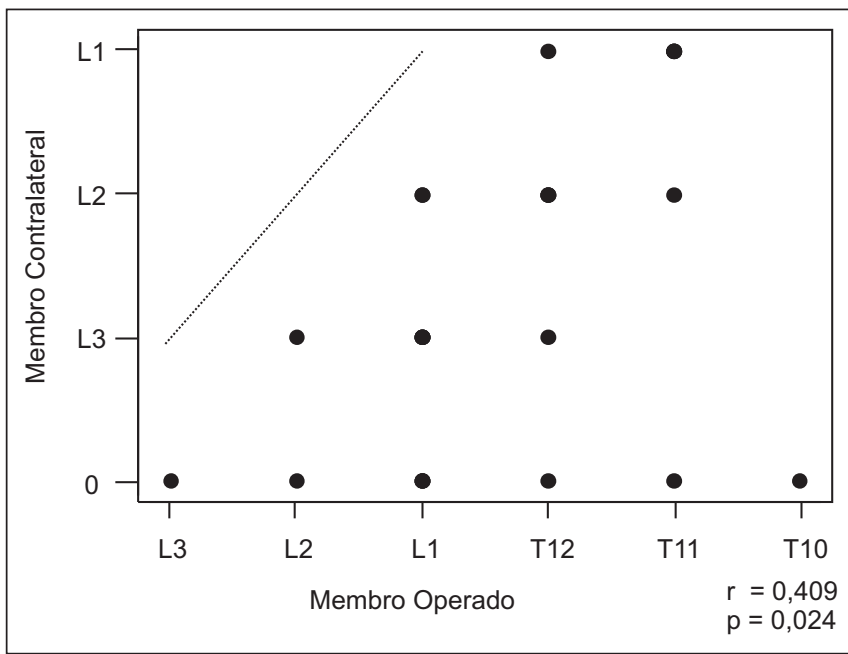

Figura 4 - Dispersão do Bloqueio Sensitivo ao Final da Cirurgia: Membro Operado Versus Membro Contralateral

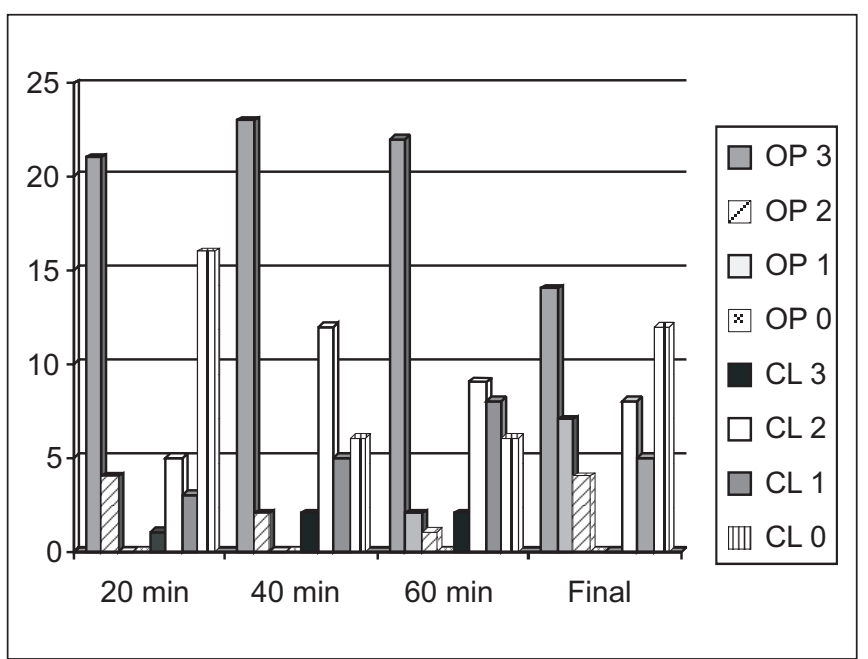

Figura 5 - Bloqueio Motor no Lado Operado e Contralateral nos Diferentes Tempos 
Em todos os pacientes a raquianestesia foi satisfatória para o procedimento e nenhum paciente necessitou de complementação com anestesia geral. Um paciente referiu dor ao torniquete aos 65 minutos de cirurgia. Dois pacientes dos 6 que não apresentaram qualquer bloqueio no membro contralateral referiram perda de propiocepção. Não ocorreram alterações da pressão arterial e da freqüência cardíaca em nenhum paciente. Não se observou retenção urinária nos pacientes estudados e nenhum paciente desenvolveu cefaléia pós-raquianestesia. Não houve relato de dor nas costas, nádegas ou pernas que pudessem ser classificados como SNT, nem ocorreram complicações neurológicas tardias.

Não ocorreu falha da anestesia. Todos os pacientes ficaram satisfeitos com a técnica. Os 6 pacientes que apresentaram raquianestesia unilateral relataram sensação agradável em permanecer sentindo o membro contralateral.

\section{DISCUSSÃO}

Baixas doses de bupivacaína isobárica $(5 \mathrm{mg})$ na velocidade de $1 \mathrm{ml} .30 \mathrm{~s}^{-1}$ através de agulha $27 \mathrm{G}$ Quincke produziram bloqueio unilateral completo em $64 \%$ dos pacientes aos 20 minutos que evoluiu para apenas $28 \%$ aos 40 minutos, mostrando que a solução isobárica de bupivacaína mobiliza-se no LCR até este momento. A raquianestesia unilateral demonstrou ser segura e efetiva em cirurgias ortopédicas de um membro, reduzindo o impacto hemodinâmico dos bloqueios realizados no neuro-eixo ${ }^{1,2,8}$ e aumentando a satisfação dos pacientes com a técnica.

A densidade da bupivacaína a $0,5 \%$ a $37^{\circ} \mathrm{C}$ é $0,9993 \mathrm{~g} \cdot \mathrm{ml}^{-1} 9$. Por definição, a baricidade é a relação entre a densidade da solução injetada e a densidade do LCR. A densidade média do LCR é de 1,00059 $\pm 0,00020 \mathrm{~g} \cdot \mathrm{ml}^{-1} 10$. A densidade da bupivacaína a $0,5 \%$ é ligeiramente hipobárica.

A utilização da raquianestesia unilateral tem aumentado bastante nos últimos anos, por produzir bloqueio motor de menor duração e em apenas um membro, proporcionando conforto do paciente, além de evitar as alterações hemodinâmicas. A bupivacaína a $0,5 \%$ isobárica é um anestésico local de longa duração de ação e normalmente emprega-se em sobredose para cirurgias ortopédicas de curta duração. Adose de $5 \mathrm{mg}$ de bupivacaína a $0,5 \%$ isobárica proporcionou um tempo de recuperação de 2,15 horas no membro operado e 1,65 hora no membro contralateral, menor que 2,93 horas obtido com $6 \mathrm{mg}$ de bupivacaína a $0,5 \%$ isobárica $^{7}$, e menor do que 2,32 horas com a mesma dose de bupivacaína a $0,15 \%$ hipobárica ${ }^{3}$.

Diversos autores preferem a agulha ponta de lápis por direcionar o fluxo do anestésico com objetivo de produzir raquianestesia unilateral ${ }^{1,6,7,11-13}$. Entretanto, outros ${ }^{2,14-16}$ preferem a agulha ponta cortante (Quincke) pelo potencial perigo da injeção lenta em sentido caudal proporcionar altas concentrações de anestésicos hiperbáricos. A agulha tipo Quincke proporciona rápido aparecimento do $\operatorname{LCR}^{17}$, não causa má distribuição do anestésico local ${ }^{18}$ e cursa com baixa incidência de cefaléia ${ }^{19}$. O tempo médio para atingir o nível máximo de analgesia com $5 \mathrm{ml}$ de bupivacaína a $0,5 \%$ foi de 17,3 minutos ${ }^{20}$. O nível da analgesia da bupivacaína a 0,5\% isobárica aumenta acima de 4 segmentos quando o paciente é colocado em cefaloaclive de $30^{\circ}$ por 80 a 115 minutos ${ }^{21}$. Neste estudo os pacientes permaneceram na posição lateral por 20 minutos e com a mesa cirúrgica horizontal e o nível sensitivo no membro operado variou de $T_{8}$ a $L_{1}$ e no membro contralateral entre $T_{12}$ a $\mathrm{L}_{3}$. Em trabalho com $5 \mathrm{mg}$ de bupivacaína hipobárica e permanecendo pelos mesmos 20 minutos o nível variou de $\mathrm{T}_{7}$ a $\mathrm{L}_{1}{ }^{3}$.

A raquianestesia unilateral pode ser realizada com soluções hipobáricas, isobáricas e hiperbáricas e a incidência de raquianestesia unilateral varia de um trabalho para outro. Com soluções isobáricas a incidência é de $37 \%{ }^{6}$, maior dos que os $28 \%$ obtido neste trabalho.

Um dos objetivos da raquianestesia unilateral é a diminuição da hipotensão arterial que pode ocorrer com a raquianestesia, fato confirmado quando se comparou a raquianestesia convencional com a raquianestesia assimétrica ${ }^{1}$. O uso de soluções hiperbáricas para obtenção de raquianestesia unilateral proporciona incidência de $10 \%{ }^{15}$ a $20 \%{ }^{12}$ de hipotensão arterial independente da velocidade de injeção. Já a utilização de soluções hipobáricas ${ }^{2,6,22,23}$ resulta numa menor incidência. Com soluções isobáricas a incidência foi $5 \%{ }^{6} \mathrm{e}$ $6,7 \%{ }^{7}$, resultados maiores dos que o do presente trabalho onde não se observou nenhum caso de hipotensão arterial.

A regressão de dois segmentos com baixas doses de bupivacaína a $0,5 \%$ hiperbárica oscila entre $67^{1}$ e $99^{18}$ minutos. Já com o uso de bupivacaína hipobárica a regressão ocorreu em 53 minutos e a liberação do paciente entre 180 e 190 minutos ${ }^{24}$. No presente estudo não foi avaliada a regressão de dois segmentos, mas sim o desaparecimento completo do bloqueio que variou de 110 a 180 minutos no membro operado e 95 a 155 minutos no membro contralateral. Desta forma, o uso de $5 \mathrm{mg}$ de bupivacaína isobárica pode ser uma boa indicação para cirurgia ambulatorial.

Agulha ponta de lápis e injeção lenta são fatores que contribuem para o aparecimento de sintomas neurológicos transitórios, principalmente quando lidocaína hiperbárica é utiliza$\mathrm{da}^{25}$. Usando agulha ponta cortante e bupivacaína isobárica nenhum caso de sintomas neurológicos foi observado neste estudo, que está de acordo com outros autores ${ }^{26,27}$ que avaliaram a influência da velocidade da injeção de bupivacaína hiperbárica a $0,5 \%$ em distribuição lateral.

A agulha ponta cortante cursa com uma baixa incidência de cefaléia $^{18}$, fato confirmado neste estudo em que não ocorreu nenhum caso de cefaléia, da mesma forma em estudo anterior $\mathrm{com}^{2}$ e sem ${ }^{3}$ retirada de 3 a $5 \mathrm{ml}$ de LCR.

A permanência de 20 minutos em decúbito lateral pode ser um tempo precioso na prática da anestesia ambulatorial, mas esta desvantagem pode ser minimizada pelas vantagens. Quando altas doses de bupivacaína isobárica ${ }^{28}$ ou hiperbárica ${ }^{29}$ são usadas, o nível e a unilateralidade da anestesia pode mudar após movimento do paciente entre 60 a 120 minutos após a injeção. Este trabalho confirma que baixas doses das soluções isobáricas se mobilizam após 20 minutos dentro do LCR.

Concluindo, este estudo demonstrou que a raquianestesia unilateral com $5 \mathrm{mg}$ de bupivacaína a $0,5 \%$ isobárica resulta num bloqueio motor intenso com regressão total de $135 \mathrm{mi}-$ nutos no lado operado, 99 minutos no lado não operado. Asolução isobárica de bupivacaína se mobiliza dentro do LCR 
após 20 minutos, resultando num bloqueio unilateral em apenas $28 \%$ dos pacientes. A principal vantagem do uso da raquianestesia unilateral com $5 \mathrm{mg}$ de bupivacaína isobárica é a grande estabilidade cardiocirculatória e sua regressão rápida, podendo ser uma nova opção para cirurgia ambulatorial.

\section{AGRADECIMENTO}

Agradecemos ao Prof. José Antônio Cordeiro pela valiosa orientação no estudo estatístico.

\section{Low Isobaric 0.5\% Bupivacaine Doses for Unilateral Spinal Anesthesia}

Luiz Eduardo Imbelloni, TSA, M.D.; Lúcia Beato, TSA, M.D.; M.A. Gouveia, TSA, M.D.

\section{INTRODUCTION}

Anesthesiologists are often faced with unilateral surgical procedures on one lower limb, especially during outpatient and orthopedic procedures. In such situations, unilateral spinal anesthesia is advantageous over conventional spinal anesthesia. These advantages include decreased hypotension during spinal anesthesia ${ }^{1-3}$, faster anesthetic recovery ${ }^{2,3}$ and increased patients satisfaction ${ }^{2,3}$. Lateral position, low anesthetic doses, pencil point needles and slow injection rate have been suggested to enhance unilateral spinal anesthesia ${ }^{4,5}$. Theoretically non-isobaric local anesthetic injections may induce unilateral block in patients in the lateral position. Isobaric bupivacaine solutions were compared to hyperbaric ${ }^{6}$ and hypobaric ${ }^{7}$ solutions to induce unilateral spinal anesthesia. Our study aimed at evaluating the attempt to asymmetrically distribute blockade between the operated and contralateral limb and its influence in the incidence of arterial hypotension during spinal anesthesia for lower limb orthopedic procedures with low doses of isobaric $0.5 \%$ bupivacaine.

\section{METHODS}

After the Clinics Disclosure and Publication Board of Directors approval and their informed consent, participated in this prospective study 25 patient's physical status ASA I and II, aged 21 to 67 years, submitted to elective and urgency orthopedic surgeries involving just one limb. Exclusion criteria were hypovolemia, coagulation disorders, infection and refusal of the method. Monitoring included noninvasive blood pressure, heart rate and pulse oximetry. Patients were not premedicated. Venous access was installed in the operating room with lactated Ringer's solution and 25 to $50 \mu \mathrm{g}$ fentanyl were injected.

Patients were placed in the lateral position with the limb to be operated facing upwards. Spine was horizontally positioned before spinal puncture and the table was leveled in the horizontal position (zero) throughout the procedure. Paramedian spinal puncture was performed at the $L_{3}-L_{4}$ interspace with $27 \mathrm{G}$ Quincke needle (B. Braun) and $5 \mathrm{mg}$ isobaric $0.5 \%$ bupivacaine were injected at the rate of $1 \mathrm{~mL} .30 \mathrm{~s}^{-1}$ with needle tip oriented upwards. Patients were maintained in this position for 20 minutes and then placed in the supine position. Sensory block was evaluated by loss of sensation to cold (ice) and to a $27 \mathrm{G}$ needle pinprick, while motor block was evaluated by Bromage's modified scale $(0=$ no motor block to $3=$ total motor block). Sensory and motor blocks were evaluated in both limbs at 20,40 and 60 minutes, and at the end of the surgery. Hemodynamic parameters were evaluated at 3-minute intervals during the first 10 minutes after spinal puncture and then at 5-minute intervals until the end of the surgery. Arterial hypotension was defined as a $30 \%$ decrement from baseline values, while bradycardia was defined as HR below $50 \mathrm{bpm}$. Arterial hypotension was treated with $1 \mathrm{mg}$ boluses of ethylphenylephrine i.v. and bradycardia with $0.5 \mathrm{mg}$ atropine. During surgery, a pneumatic tourniquet was inflated $100 \mathrm{mmHg}$ above systolic blood pressure on the operated limb. Oxygen ( 2 L. min $^{-1}$ ) was administered through nasal catheter or Hudson's mask. Midazolam (1 to $2 \mathrm{mg}$ ) was administered after the second evaluation (40 minutes).

Patients were followed until the $3^{\text {rd }}$ postoperative day for information about their satisfaction with the technique, post-dural puncture headache or transient neurological symptoms (TNS), and until the $30^{\text {th }}$ day for permanent neurological complications.

Quantitative variables were analyzed in terms of descriptive statistics with means \pm standard deviation, minimum, maximum and median - when this was the best alternative. Blockades on both limbs were compared by non-parametric sign test, and correlations were evaluated by Pearson's correlation coefficient. Significance level was $5 \%$.

\section{RESULTS}

Demographics data are shown in table I.

Unilateral block was induced in $64 \%$ of patients at 20 minutes. At 40 and 60 minutes, just $28 \%$ of patients remained with unilateral block. At the end of the surgery, $32 \%$ of patients presented unilateral block. Total blockade regression time was $2.34 \pm 0.33 \mathrm{~h}$ on the operated limb and $1.64 \pm 0.67 \mathrm{~h}$ on the contralateral limb. There were no significant differences in total blockade regression according to gender $(p=0.95$, operated; $p=0.97$, contralateral), or to surgery duration $(p=0.86)$. Females were older as compared to males $(p=0.028)$.

Sensory block spread in the operated limb varied from $T_{8}$ through $L_{1}$ and on the contralateral limb from $T_{12}$ through $L_{3}$, showing a clear between-limb dissociation. At 20 minutes, 9 patients exhibited sensory block on the contralateral limb; at 40 minutes 18 patients, and at 60 minutes 17 patients exhibited sensory block on the contralateral limb. Unilateral blocks occurred in 7 patients $(28 \%)$ while blocks were bilateral in the remaining 18 patients $(72 \%)$. Blocks were significantly higher on operated limbs $(p<0.0001)$, as shown on figures 1 through 4. There was linear correlation between sensory block spread in both limbs, as shown in figures 1 to 4 . 
Table I - Demographics Data, Surgery and Sensory Block Duration

\begin{tabular}{lc}
\hline Age $($ years) * & $39.11 \pm 13.75$ \\
Female & $43.90 \pm 14.50$ \\
Male & $33.40 \pm 11.40$ \\
Weight $(\mathrm{kg}){ }^{*}$ & $67.12 \pm 13.59$ \\
Height $(\mathrm{cm}){ }^{*}$ & $163.44 \pm 6.60$ \\
Gender & 14 \\
$\quad$ Female & 11 \\
$\quad$ Male & $1.33 \pm 0.19$ \\
Surgery duration $(\mathrm{h}){ }^{*}$ & \\
Blockade duration $(\mathrm{h}){ }^{*}$ & $2.34 \pm 0.33$ \\
Operated limb & $1.64 \pm 0.67$ \\
Contralateral limb & \\
\hline
\end{tabular}

* Values expressed in Mean \pm SD

At 20 minutes, total motor block (grade 3 ) of the operated limb was noted in 21 patients, and grade 2 blocks occurred in 4 patients. At 40 minutes, two additional patients exhibited total motor block. Total motor block of the contralateral limb occurred in 1 patient, grade 2 in 5 , grade 1 in 3 and no motor block in 16 patients, at 20 minutes. At 40 minutes, total motor block was noted in 2 patients, grade 2 in 12, grade 1 in 5, and no motor block in 6 patients (Figure 5 ). Unilateral motor block was present in 16 patients $(64 \%)$ at 20 minutes and in 6 patients $(24 \%)$ at 40 minutes. Motor block on the operated limb was higher as compared to the contralateral limb in all evaluations $(p<0.0001)$.

Spinal anesthesia was satisfactory, as no patient needed general anesthesia complementation. One patient referred tourniquet pain after 65 minutes of surgery. Two out of the 6 patients without contralateral limb blockade have referred loss of proprioception. No blood pressure and or heart rate changes were noted. No urinary retention was observed and no patient developed post-dural puncture headache. No back, buttock or leg pain has been referred that could be classified as TNS. No late neurological complications occurred.

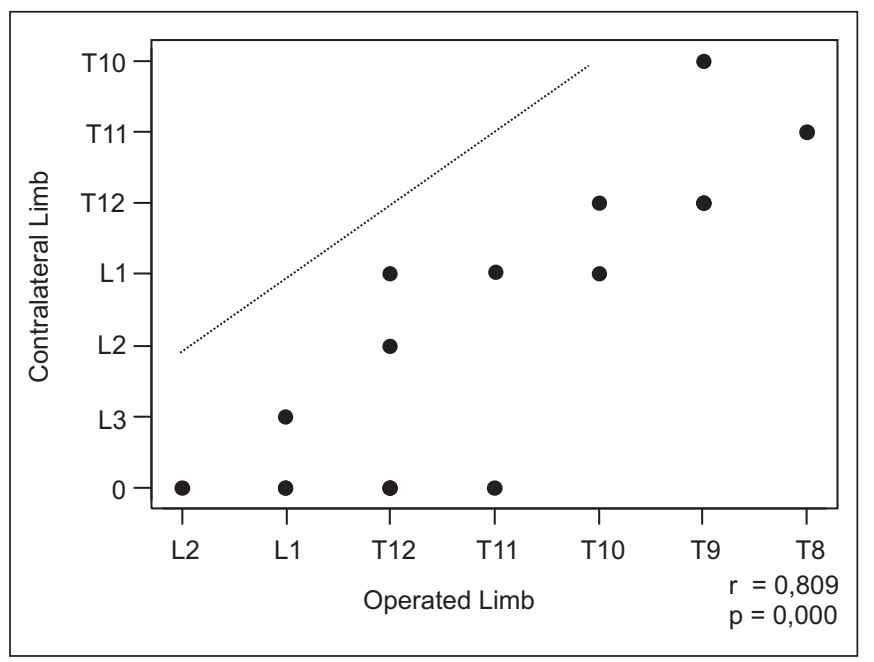

Figure 1 - Sensory Block Spread at 20 Minutes: Operated Limb Versus Contralateral Limb

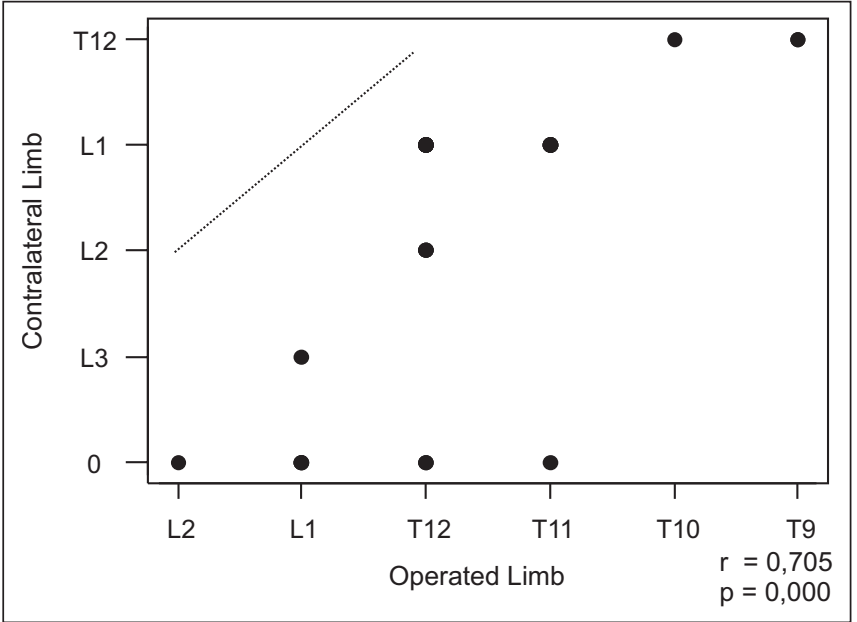

Figure 2 - Sensory Block Spread at 40 Minutes: Operated Limb Versus Contralateral Limb

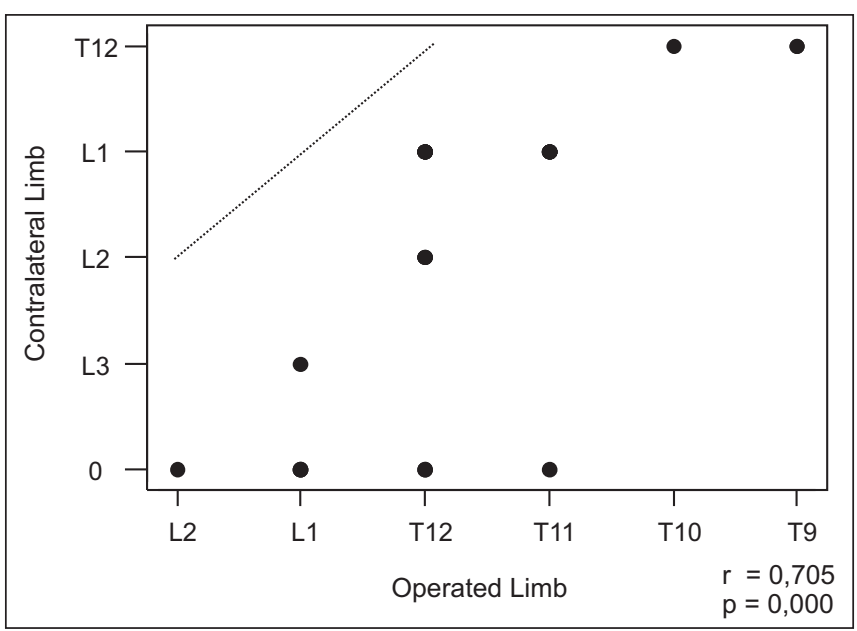

Figure 3 - Sensory Block Spread at 60 Minutes: Operated Limb Versus Contralateral Limb

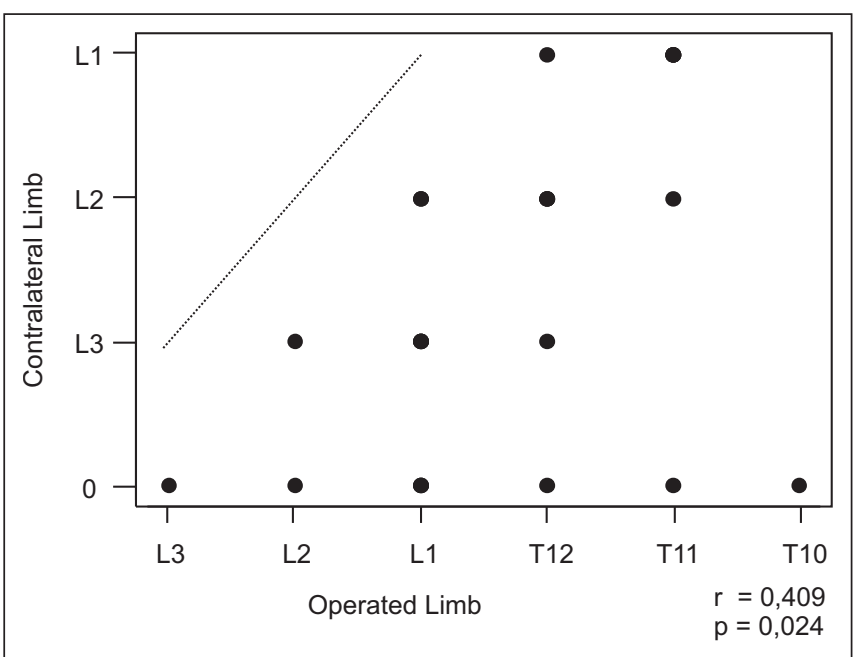

Figure 4 - Sensory Block Spread at Surgery Completion: Operated Limb Versus Contralateral Limb

Revista Brasileira de Anestesiologia Vol. 54, № 3, Maio - Junho, 2004 


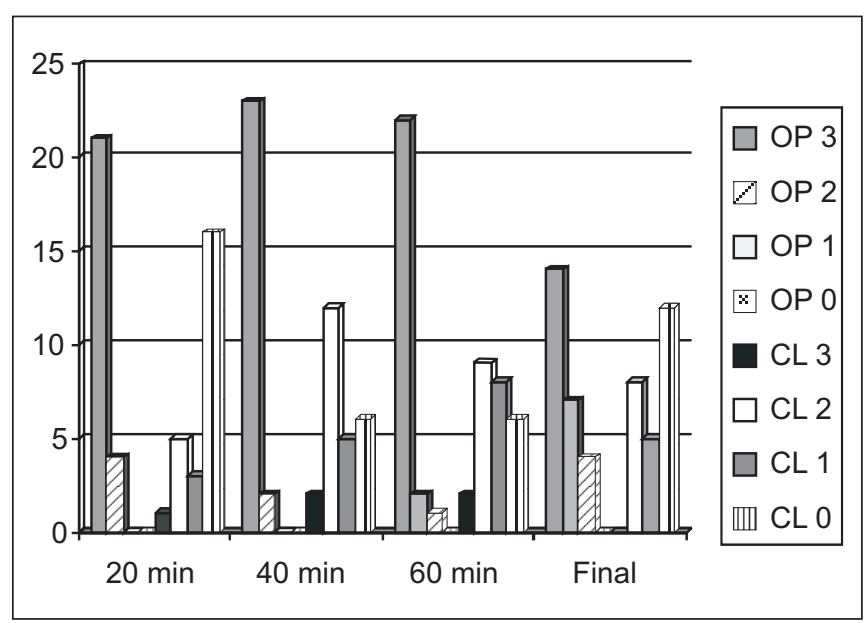

Figure 5 - Block Operated Limb versus Contralateral Limb in Differents Times

All patients were satisfied with the technique. All 6 patients with unilateral spinal anesthesia referred a nice sensation of being able to feel the contralateral limb.

\section{DISCUSSION}

Low doses of isobaric bupivacaine $(5 \mathrm{mg})$ at the rate of 1 $\mathrm{mL} .30 \mathrm{~s}^{-1}$ through $27 \mathrm{G}$ Quincke needle induced total unilateral block in $64 \%$ of patients at 20 minutes, but this figure evolved to just $28 \%$ at 40 minutes, showing that isobaric bupivacaine moves in the CSF in this time-frame. Unilateral spinal anesthesia has shown to be safe for single limb orthopedic procedures, decreasing the hemodynamic impact of neuraxial blocks ${ }^{1,2,8}$ and increasing patients' satisfaction with the technique.

The density of $0.5 \%$ bupivacaine at $37^{\circ} \mathrm{C}$ is $0.9993 \mathrm{~g} \cdot \mathrm{mL}^{-1}$. By definition, baricity is the ratio between injected solution density and CSF density. Mean CSF density is $1.00059 \pm$ $0.00020 \mathrm{~g} \cdot \mathrm{mL}^{-110}$. The density of $0.5 \%$ bupivacaine is slightly hypobaric.

Unilateral spinal anesthesia has been increasingly used in recent years for inducing unilateral short-lasting motor block, allowing patients' comfort and preventing hemodynamic changes. Isobaric $0.5 \%$ bupivacaine is a long-lasting local anesthetic, which is usually overdosed during short orthopedic procedures. The dose of $5 \mathrm{mg}$ of isobaric $0.5 \%$ bupivacaine has allowed a recovery time of 2.15 hours in the operated limb and of 1.65 hours in the contralateral limb, shorter than 2.93 hours obtained with $6 \mathrm{mg}$ isobaric $0.5 \%$ bupivacaine $^{7}$, and shorter than the 2.32 -hour duration with the same dose of hypobaric $0.15 \%$ bupivacaine ${ }^{3}$.

Several authors prefer pencil point needles for directing anesthetic flow to induce unilateral spinal anesthesia ${ }^{1,6,7,11-13}$. Others $^{2,14-16}$, however, prefer cutting-tip needles (Quincke) due to the potential hazard of slow caudal injections promoting high hyperbaric anesthetic concentrations. Quincke needles allow for fast CSF flow ${ }^{17}$, do not promote poor local anesthetic distribution ${ }^{18}$ and are associated with low incidence of headache ${ }^{19}$.
Mean time to reach maximal level of analgesia after $5 \mathrm{~mL}$ of $0.5 \%$ plain bupivacaine has been shown to be 17.3 minutes ${ }^{20}$. Upper sensory level increases more than four dermatomes when patients are positioned in $30^{\circ}$ head-up position for 80 to 115 minutes ${ }^{21}$. In this study, patients remained in horizontal lateral decubitus for 20 minutes and the sensory level on the operated limb ranged from $T_{8}$ to $L_{1}$, while on the contralateral limb sensory block ranged from $T_{12}$ to $L_{3}$. In a study in which 5 $\mathrm{mg}$ hypobaric bupivacaine was used and patients remaining in lateral position for the same 20 minutes, upper sensory level varied from $T_{7}$ through $L_{1}{ }^{3}$.

Unilateral spinal anesthesia may be induced with hypobaric, isobaric, or hyperbaric solutions and the incidence of unilateral spinal anesthesia varies according to the study. With isobaric solutions the incidence is $37 \%{ }^{6}$, or higher than our $28 \%$.

One objective of unilateral spinal anesthesia is to decrease hypotension, which may happen during spinal anesthesia, a fact that was confirmed when conventional anesthesia was compared to asymmetric anesthesia ${ }^{1}$. Hyperbaric solutions for unilateral spinal anesthesia are associated with an incidence of $10 \%{ }^{15}$ to $20 \%{ }^{12}$ of arterial hypotension, regardless of injection rate. Hypobaric solutions ${ }^{2,6,22,23}$ result in lower incidence. With isobaric solutions, the incidence has been of $5 \%{ }^{6}$ and $6.7 \%{ }^{7}$, higher results than those found in our study where no arterial hypotension episode was observed.

The regression of two segments with low doses of hyperbaric $0.5 \%$ bupivacaine varies from $67^{1}$ to $99^{18}$ minutes. With hypobaric bupivacaine, regression was present at $53 \mathrm{~min}-$ utes and patients were released after 180 to 190 minutes postoperatively ${ }^{24}$. Two segments regression was not evaluated in our study, but rather total blockade regression, which varied from 110 to 180 minutes in the operated limb and from 95 to 155 minutes in the contralateral limb, what suggests that $5 \mathrm{mg}$ isobaric bupivacaine may be a good indication for outpatient procedures.

Pencil point needle and slow injection rate may contribute for the occurrence of transient neurological symptoms, especially when hyperbaric lidocaine is used ${ }^{25}$. No neurological symptoms have been observed in our study with cutting point needles and bupivacaine, in agreement with other authors ${ }^{26,27}$ who have evaluated the influence of hyperbaric $0.5 \%$ bupivacaine injection rate on lateral distribution.

Cutting point needles are associated with low incidence of headache ${ }^{18}$, which was confirmed in our study where no headache has been observed, similarly to a previous study with ${ }^{2}$ and without ${ }^{3}$ removal of 3 to $5 \mathrm{~mL}$ CSF.

The maintenance of the lateral position for 20 minutes may be a precious time for outpatient anesthetic practice, but this disadvantage may be overcome by advantages. When high isobaric ${ }^{28}$ or hyperbaric ${ }^{29}$ bupivacaine doses are used, anesthetic depth and unilaterality may change after moving the patient 60 to 120 minutes after injection. Our study has confirmed that low isobaric solution doses move after 20 minutes in the CSF. In conclusion, this study has shown that unilateral spinal anesthesia with $5 \mathrm{mg}$ isobaric $0.5 \%$ bupivacaine results in intense motor block with total regression in 115 minutes on the operated side and in 99 minutes on the contralateral side. Iso- 
baric bupivacaine moves in the CSF after 20 minutes resulting in unilateral block in just $28 \%$ of patients. Major advantages of unilateral spinal anesthesia with $5 \mathrm{mg}$ isobaric bupivacaine are its cardiocirculatory stability and fast regression, and it might be a new alternative for outpatient procedures.

\section{ACKNOWLEDGEMENT}

We acknowledge Prof. José Antonio Cordeiro for his valuable guidance during statistical analysis.

\section{REFERÊNCIAS - REFERENCES}

01. Casati A, Fanelli G, Aldegheri G et al - Frequency of hypotension during conventional or asymmetric hyperbaric spinal block. Reg Anesth Pain Med, 1999;24:214-219.

02. Imbelloni LE, Beato L, Gouveia MA - Unilateral spinal anesthesia with hypobaric bupivacaine. Rev Bras Anestesiol, 2002;52:27-33

03. Imbelloni LE, Beato L, Gouveia MA - A low dose of hypobaric bupivacaine for unilateral spinal anesthesia. Rev Bras Anestesiol, 2003;53:579-585.

04. Tanasichuk MA, Schultz EA, Matthews JH et al - Spinal hemianalgesia: an evaluation of a method, its applicability, and influence of the incidence of hypotension. Anesthesiology, 1961;22: 74-85.

05. Casati A, Fanelli G, Cappelleri G et al - Low dose hyperbaric bupivacaine for unilateral spinal anaesthesia. Can J Anaesth, 1998;45:850-854

06. Kuusniemi KS, Pihlajamäki KK, Pitkänen MT - A low dose of plain or hyperbaric bupivacaine for unilateral spinal anesthesia. Reg Anesth Pain Med, 2000;25:605-610.

07. Kuusniemi KS, Pihlajamäki KK, Pitkänen MT et al - Low-dose bupivacaine: a comparison of hypobaric and near isobaric solutions for arthroscopic surgery of the knee. Anaesthesia, 1999;54 540-545.

08. Casati A, Fanelli G, Berti M et al - Cardiac performance during unilateral lumbar spinal block after crystalloid preload. Can J Anaesth, 1997;44:623-628.

09. Cangiani LM - Determinação da densidade e da baricidade das misturas para anestesia subaracnóidea. Rev Bras Anestesiol, 2000;50:92-94.

10. Lui AC, Polis TZ, Cicutti NJ - Densities of cerebrospinal fluid and spinal anaesthetic solutions in surgical patients at body temperature Can J Anesth, 1998:45:297-303.

11. Enk D, Prien T, Van Aken $\mathrm{H}$ et al - Success rate of unilateral spinal anesthesia is dependent on injection flow. Reg Anesth Pain Med, 2001;26:420-427

12. Casati A, Fanelli G, Cappelleri G et al - Effects of speed intrathecal injection on unilateral spinal block by $1 \%$ hyperbaric bupivacaine. $A$ randomized, double-blind study. Minerva Anestesiol, 1999;65:5-10.

13. Fanelli G, Borghi B, Casati A et al - Unilateral bupivacaine spinal anesthesia for outpatient knee arthroscopy. Can J Anaesth, 2000:47:746-751.

14. Gouveia MA, Labrunie GM - Raquianestesia hipobárica com bupivacaína 0,15\%. Rev Bras Anestesiol, 1985;35:519-521.

15. Esmaoglu A, Boyaci A, Ersoy Ö et al - Unilateral spinal anaesthesia with hyperbaric bupivacaine. Acta Anaesthesiol Scand 1998;42:1083-1087.

16. Iselin-Chaves I, Van Gessel EF, Donald F et al - The effects of solution concentration and epinephrine on lateral distribution of hyperbaric tetracaine spinal anesthesia. Anesth Analg, 1996;83:755-759.

17. Imbelloni LE, Carneiro ANG, Sobral MGC - Tempo de gotejamento de líquido cefalorraquidiano com agulhas espinhais de fino calibre. Rev Bras Anestesiol, 1995:45: 155-158.

18. Holman SJ, Robinson RA, Beardsley D et al - Hyperbaric dye solution distribution characteristics after pencil-point needle injection in a spinal cord model. Anesthesiology, 1997;86: 966-973.

19. Imbelloni LE, Sobral MGC, Carneiro ANG - Cefaléia pós-raquianestesia e o desenho das agulhas. Experiência com 5050 casos. Rev Bras Anestesiol, 2001;51:43-52.
20. Cameron AE, Arnold RW, Ghoris MW et al - Spinal analgesia using bupivacaína $0.5 \%$ plain. Anaesthesia, 1981;36:318-322.

21. Niemi L, Tuominen M, Pitkänen M et al - Effect of late posture change on the level of spinal anaesthesia with plain bupivacaine. $\mathrm{Br}$ J Anaesth, 1993;71:807-809.

22. Kuusniemi KS, Pihlajamäki KK, Pitkänen MT et al - A low-dose hypobaric bupivacaine spinal anesthesia for knee arthroscopies. Reg Anesth, 1997:22:534-538.

23. Kuusniemi KS, Pihlajamäki KK, Kirvelä OA et al - Spinal anesthesia with hypobaric bupivacaine for knee arthroscopies: effect of posture on motor block. Reg Anesth Pain Med, 2001;26:30-34

24. Ben-David B, Levin H, Solomon E et al - Spinal bupivacaine in ambulatory surgery: the effect of saline dilution. Anesth Analg, 1996;83:716-720.

25. Beardsley D, Holman S, Gantt R et al - Transient neurologic deficit after spinal anesthesia: local anesthetic maldistribution with pencil point needles? Anesth Analg, 1995;81:314-320.

26. Pittoni G, Toffoletto F, Calcarella G et al - Spinal anesthesia in outpatient knee surgery: 22-gauge versus 25-gauge Sprotte needle. Anesth Analg, 1995;81:73-79.

27. Casati A, Fanelli G, Cappelleri G et al - Does speed of intrathecal injection affect the distribution of $0.5 \%$ hyperbaric bupivacaine? $\mathrm{Br} \mathrm{J}$ Anaesth, 1998;81:355-357.

28. Niemi L, Tuominen M, Pitakänen $M$ et al - Effect of late posture change on the level of spinal anaesthesia with plain spinal bupivacaine. Br J Anaesth, 1993;71:807-809

29. Povey HM, Jacobsen J, Westergaard-Nielsen J - Subarachnoid analgesia with hyperbaric $0.5 \%$ bupivacaine: effect of a 60 -min period sitting. Acta Anaesthesiol Scand, 1989;33:295-297

\section{RESUMEN}

Imbelloni LE, Beato L, Gouveia MA - Bajas Dosis de Bupivacaína a $0,5 \%$ Isobárica para Raquianestesia Unilateral

JUSTIFICATIVA Y OBJETIVOS: La raquianestesia unilateral puede presentar diversas ventajas, principalmente en el paciente ambulatorial. Bajas dosis de anestésicos locales, lenta velocidad de inyección subaracnóidea y posición de decúbito lateral son relacionados con la facilidad de producir distribución unilateral en la raquianestesia. En este estudio, fue verificada la posibilidad de obtenerse raquianestesia unilateral con bupivacaína a 0,5\% isobárica.

MÉTODO: La raquianestesia con $1 \mathrm{ml}$ de bupivacaína isobárica a $0,5 \%$ (5 mg) fue realizada a través de aguja 27G Quincke en 25 pacientes, estado físico ASA / y I/ sometidos a cirugías ortopédicas. La punción subaracnóidea fue realizada por vía lateral con el paciente en decúbito lateral, con el miembro a ser operado vuelto para arriba, y $1 \mathrm{ml}$ de bupivacaína hipobárica fue inyectado en la velocidad de $1 \mathrm{ml} 30 \mathrm{~s}^{-1}$. Bloqueos sensitivo y motor (picada de aguja y escala de 0 a 3) fueron comparados entre el lado a ser operado y el contralateral.

RESULTADOS: Los bloqueos motor y sensitivo entre el lado operado y el contralateral fueron significativamente diferentes en todos los tiempos evaluados. En el miembro operado todos los pacientes presentaran anestesia quirúrgica. En el miembro contralateral, a los 20 minutos, 9 pacientes presentaron bloqueo sensitivo; a los 40 minutos 18 pacientes y a los 60 minutos 17 pacientes. Por tanto, el bloqueo sensitivo unilateral ocurrió en 7 pacientes (28\%) y en ambos miembros en 18 pacientes $(72 \%)$. Raquianestesia unilateral fue obtenida en $28 \%$ de los pacientes. Estabilidad hemodinámica fue observada en todos los pacientes. Ningún paciente desenvolvió cefalea pós-raquianestesia.

CONCLUSIONES: La bupivacaína isobárica (5 mg) proporciona predominante bloqueo unilateral después de 20 minutos en la posición lateral. La solución isobárica de bupivacaína se moviliza dentro del LCR después de 20 minutos, resultando en un bloqueo unilateral en apenas $28 \%$ de los pacientes. La principal ventaja de la raquianestesia unilateral es la estabilidad hemodinámica y su rápida regresión, pudiendo ser una nueva opción para cirugía ambulatorial. 